\title{
Properties of lower hybrid waves
}

\author{
Alix L. Verdon ${ }^{1}$, I. H. Cairns ${ }^{1}$, D. B. Melrose ${ }^{1}$ and P. A. Robinson ${ }^{1}$ \\ ${ }^{1}$ School of Physics A28, \\ University of Sydney, NSW 2006, \\ Australia \\ email: a.nulsen@physics.usyd.edu.au
}

\begin{abstract}
Most treatments of lower hybrid waves include either electromagnetic or warmplasma effects, but not both. Here we compare numerical dispersion curves for lower hybrid waves with a new analytic dispersion relation that includes both warm and electromagnetic effects. Very good agreement is obtained over significant ranges in wavenumber and plasma parameters, except where ion magnetization effects become important.
\end{abstract}

Keywords. waves, plasmas, acceleration of particles, solar system: general

\section{Introduction}

Waves in heliospheric plasmas are involved in many universal heliophysical processes, including plasma heating, particle acceleration, and emission processes that are signatures of energy releases. Lower hybrid (LH) waves are of particular interest in contexts that involve both electrons and ions; these waves can transfer energy between parallel motions of electrons and perpendicular motions of ions (Omel'chenko et al. 1989; Melrose 1986; Cairns 2001; Cairns \& Zank 2002).

LH waves are nearly electrostatic with wavevectors $\mathbf{k}$ nearly perpendicular to the magnetic field, and involve oscillations of both the ions and electrons. In a cold plasma $\mathrm{LH}$ waves occur at a frequency which depends on the angle $\theta$ between the magnetic field $\mathbf{B}$ and $\mathbf{k}$ and is given by

$$
\omega^{2}=\omega_{L H}^{2}\left(1+\frac{m_{i}}{m_{e}} \cos ^{2} \theta\right) .
$$

Here $m_{e}$ and $m_{i}$ are, respectively, the masses of electrons and ions in the plasma, and the LH frequency is

$$
\omega_{L H} \approx \frac{1}{\sqrt{1 / \omega_{p i}^{2}+1 / \Omega_{e} \Omega_{i}}},
$$

where $\omega_{p i}$ is the ion plasma frequency and $\Omega_{e}$ and $\Omega_{i}$ are the electron and ion gyrofrequencies, respectively.

At the LH frequency the ions are unmagnetized and free to move across $\mathbf{B}$, but the electrons are magnetized and may only move along $\mathbf{B}$. If the wave electric field is nearly perpendicular to $\mathbf{B}$ then the electron response time is greatly increased. The LH resonance occurs only when the ion response time is less than or comparable to the electron response time; i.e. when the following is satisfied,

$$
\cos ^{2} \theta \lesssim \frac{m_{e}}{m_{i}}
$$

When warm plasma or electromagnetic effects are included in the plasma response the LH resonance becomes a propagating mode which must still satisfy equation (1.3). Hence, the components of $\mathbf{k}$ parallel and perpendicular to $\mathbf{B}$ are of such different magnitude, with 
$k_{\|} / k_{\perp} \lesssim m_{e} / m_{i} \ll 1$, that LH waves at the same frequency $\omega$ can satisfy the resonant conditions for interacting with the unmagnetized ions $\left(\omega=\mathbf{k} \cdot \mathbf{v}_{i}\right)$ and the magnetized electrons $\left(\omega=k_{\|} v_{e \|}\right)$. Thus LH waves may transfer energy from the perpendicular motions of ions to the parallel motions of electrons or vice versa, either accelerating particles or heating them.

Whenever there is a non-thermal perpendicular distribution of ions or parallel distribution of electrons, LH waves may play a role in redistributing the energy. Such distributions occur in many regions of the heliosphere, including in the outer heliosheath where pick-up ions form a ring beam distribution (Cairns \& Zank 2002), and near magnetic reconnection sites where bulk ions flows across $\mathbf{B}$ and electrons accelerated along $\mathbf{B}$ are observed (Cairns 2001), such as occur in the Earth's magnetotail and possibly in the solar corona. However, to determine where this mechanism is efficient and could be relevant we must calculate the wave growth and particle diffusion rates. To do so, we must first find the LH dispersion relation, so that we can calculate the phase and group speeds.

Section 2 introduces the existing analytic dispersion relations for LH waves, which are compared with numerical dispersion relations in Section 3. In Section 4 we compare the numerical results with a new analytic dispersion relation we have derived. Section 5 contains a discussion and summary of our results.

\section{Existing dispersion relations}

The dispersion relations most commonly used for LH waves either include electromagnetic (EM) effects and assume the plasma is cold, or include warm plasma effects and assume that the waves are electrostatic. In general when deriving a dispersion relation for LH waves it is assumed that equation (1.3) is satisfied and $\Omega_{i} \ll \omega \ll \Omega_{e} \ll \omega_{p e}$, where $\omega_{p e}$ is the electron plasma frequency, so the ions may be treated as unmagnetized and the electrons as magnetized.

Assuming the plasma is cold but including EM effects gives (Omel'chenko et al. 1989)

$$
\omega^{2} / \omega_{L H}^{2}=\frac{1}{1+\omega_{p e}^{2} / k^{2} c^{2}}\left(1+\frac{m_{i}}{m_{e}} \frac{\cos ^{2} \theta}{1+\omega_{p e}^{2} / k^{2} c^{2}}\right),
$$

where $c$ is the speed of light. This expression makes no assumption on the size of $\omega_{p e}^{2} / k^{2} c^{2}$.

Including warm plasma effects and assuming the waves are longitudinal leads to the dispersion relation (Melrose 1986)

$$
\omega^{2} / \omega_{L H}^{2}=\left(1+\frac{m_{i}}{m_{e}} \cos ^{2} \theta+\left(3 \frac{T_{i}}{T_{e}}+\frac{3}{4}\right) \frac{k^{2} V_{e}^{2}}{\Omega_{e}^{2}}\right),
$$

where $T_{e}$ and $T_{i}$ are the electron and ion temperatures, respectively, and $V_{e}$ is the electron thermal speed. This expression includes terms only to first order in $k^{2} V_{e}^{2} / \Omega_{e}^{2}$.

A third dispersion relation for LH waves was used by Bingham et al. (2002). This dispersion relation includes warm plasma effects to first order in $k^{2} V_{e}^{2} / \Omega_{e}^{2}$ and EM effects to first order in $\omega_{p e}^{2} / k^{2} c^{2}$.

\section{Comparison of numerical and existing dispersion relations}

The deviation of the LH mode frequency from the cold plasma resonance frequency [equation (1.1)] is greatest when $k^{2} V_{e}^{2} / \Omega_{e}^{2}$ is largest in the warm electrostatic approximation, and when $\omega_{p e}^{2} / k^{2} c^{2}$ is greatest in the EM cold plasma approximation. The dispersion relation used by Bingham et al. (2002) differs from equation (1.1) at both large and small 


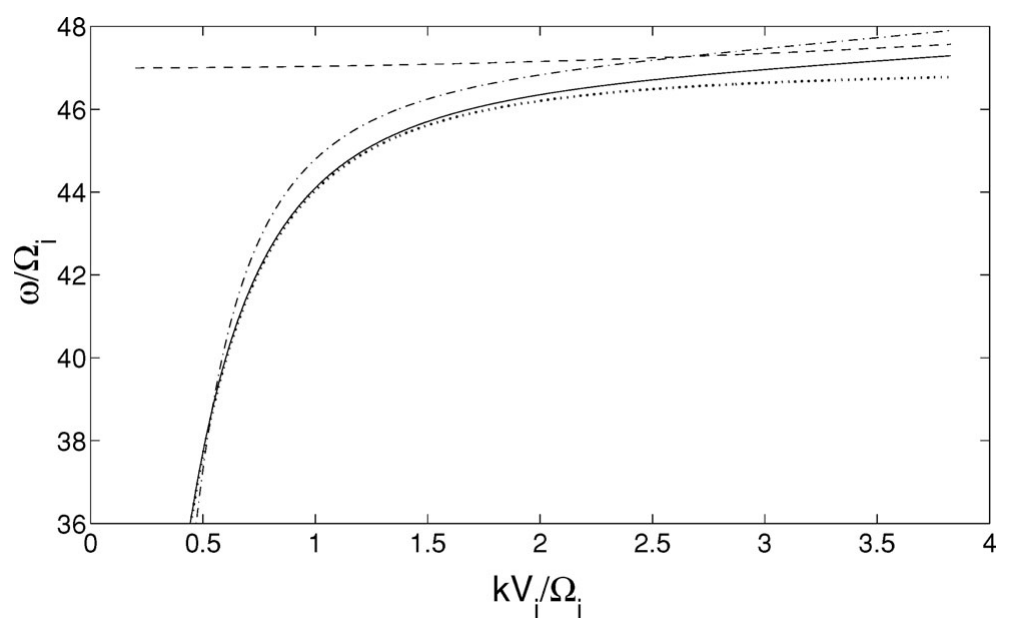

Figure 1. Warm electrostatic (dashes), cold EM (dots), Bingham et al. (2002) (dash-dot) and numerical (solid) dispersion relations for $\pi / 2-\theta=0.011 \mathrm{rad}, T_{i}=T_{e}=4000 \mathrm{~K}$ and $\omega_{p e}=10 \Omega_{e}$.

$k$. In order to determine whether any of these were appropriate we compared them with a numerical dispersion relation.

The numerical LH dispersion relation was found for some parameters using the code of Willes \& Cairns (2000). The code calculates the fully electromagnetic response tensor for the specified plasma parameters, including both electron and ion magnetization effects, and finds $\omega(\mathbf{k})$ as a root of its determinant in the complex plane. The code follows a single mode as $k$ is changed and $\theta$ is kept constant, extrapolating from previous $\omega(\mathbf{k})$.

The three existing dispersion relations introduced above are compared with the real part of the numerical dispersion relation in Fig. 1. For low $k$ the cold EM dispersion relation matches best with the numerical result. As $k$ increases the slopes differ, with the cold EM result asymptotically approaching the cold plasma resonance frequency while the numerical frequency continues to increase. Consequently the group speed for LH waves calculated in the cold EM approximation is much lower than the actual value. At higher $k$ the slope and magnitude of the warm electrostatic frequency best matches the numerical dispersion relation. Neither of these dispersion relations is adequate to describe LH waves over the range of $k$ that interact with particles with speeds around the thermal speed and slightly faster.

The Bingham et al. (2002) dispersion relation gives a better overall fit than the other two dispersion relations. Ideally, one needs a dispersion relation that allows more accurate calculations of the phase and group speed of LH waves.

\section{New results}

We derived a new analytic dispersion relation including both EM and warm effects, with terms to all orders in $\omega_{p e}^{2} / k^{2} c^{2}$ but only to first order in $k^{2} V_{e}^{2} / \Omega_{e}^{2}$. This dispersion relation will be presented elsewhere. As for the other, existing, dispersion relations we assume that equation (1.3) is satisfied and the electrons are magnetized but that the ions are not, and that there is no thermal damping of the waves. This dispersion relation reduces to equation (2.2), the warm electrostatic dispersion relation, and equation (2.1), the cold EM dispersion relation, in the appropriate limits.

A comparison of this new dispersion relation with the numerical and Bingham et al. (2002) dispersion relations is shown in Fig. 2 for a low temperature plasma. The new 


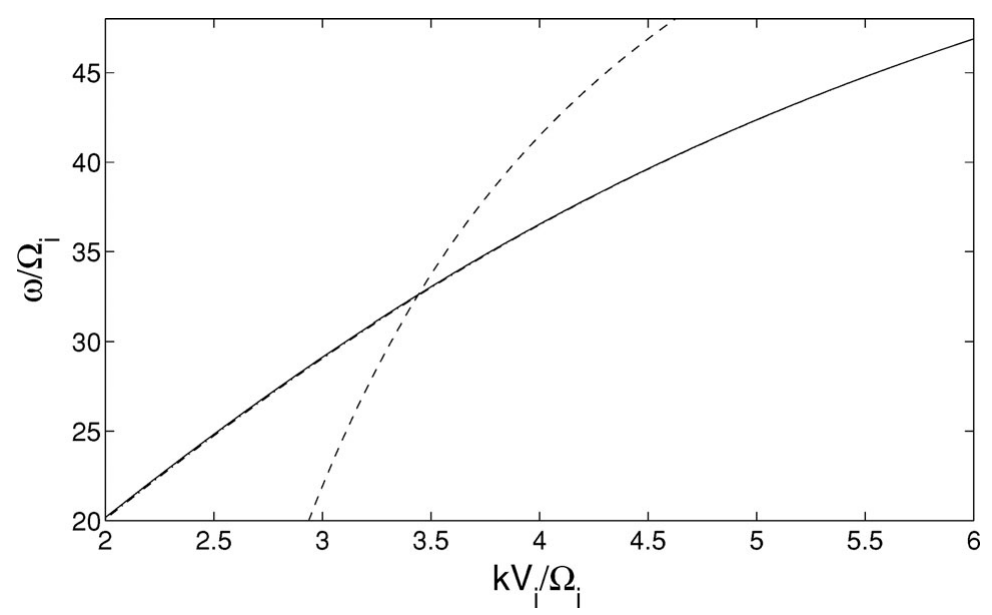

Figure 2. Bingham et al. (2002) (dashed), new analytic (dot-dashed) and numerical (solid) dispersion relations for $\pi / 2-\theta=0.024 \mathrm{rad}, T_{i}=T_{e}, T_{e}=6000 \mathrm{~K}$ and $\omega_{p e}=100 \Omega_{e}$.

analytic and numerical results are almost indistinguishable, especially for large $k$, for these plasma parameters. Our new dispersion relation matches the numerical results far more closely than the Bingham et al. (2002) dispersion relation.

A comparison of the new analytic dispersion relation with the numerical results at higher temperatures is shown in Fig. 3. In this case the agreement is not as good. At both high and low $k$ no numerical solution to the dispersion relation can be found at or near multiples of $\Omega_{i}$. As the frequency approaches a multiple of $\Omega_{i}$ the LH mode appears to break up into a series of ion Bernstein modes that make a transition between harmonics of $\Omega_{i}$ along a locus that is effectively the dispersion curve for LH waves. The analytic dispersion relation and the numerical results agree except near multiples of $\Omega_{i}$.

Increasing the ion temperature by even a small factor, with or without changing the electron temperature, decreases the range of $k$ over which numerical solutions can be found at multiple of $\Omega_{i}$. Increasing $\theta$ so that the waves are even closer to perpendicular propagation also dramatically decreases the range of $k$ over which numerical solutions at multiples of $\Omega_{i}$ can be found. There are also weaker dependencies on other parameters of the plasma at which a numerical solution for the LH wave can no longer be found at frequencies very close to multiples of $\Omega_{i}$. Similar results were found numerically, for example by Feng et al. (1992); the ion acoustic mode becomes a series of perturbations to the ion Bernstein modes for low $k$ and nearly perpendicular propagation.

\section{Discussion and Summary}

Ion magnetization effects, which are not included in any explicit analytic dispersion relation for $\mathrm{LH}$ waves, are strongest at multiples of $\Omega_{i}$. As these are included in the numerical results it is not surprising that the greatest difference between our numerical and analytic results occur at multiples of $\Omega_{i}$, and may be attributed to ion magnetization effects.

To lowest order, ion magnetization effects contribute only to the imaginary part of $\omega$, $\operatorname{Im}(\omega)$, and not to the real part, $\operatorname{Re}(\omega)$. All the numerical results shown here are only for the real part of the complex frequency. In all cases $|\operatorname{Im}(\omega)| \ll \operatorname{Re}(\omega)$, so the mode is not heavily damped and the weak damping approximation is valid. Using this approximation to include the ion magnetization effects analytically to lowest order we reproduce the same 


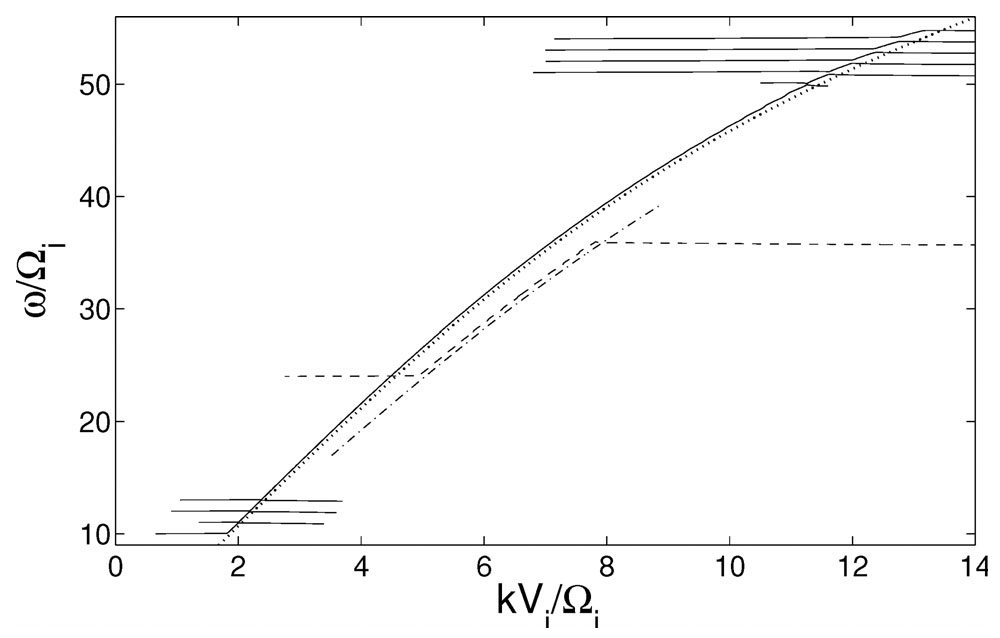

Figure 3. New analytic and numerical dispersion relations for $\omega_{p e}=100 \Omega_{e}, \pi / 2-\theta=0.024 \mathrm{rad}$, $T_{e}=24000 \mathrm{~K}$ and $T_{e}=T_{i}$ (dotted - analytic, and solid - numerical) and $T_{e}=0.8 T_{i}$ (dash-dot - analytic, and dashed - numerical).

features as the numerical results, but there are significant quantitative differences. Both numerical and analytic results show that $|\operatorname{Im}(\omega)|$ is greatest when $\operatorname{Re}(\omega)$ is a multiple of $\Omega_{i}$ and that $|\operatorname{Im}(\omega)|$ at local maxima increases from a minimum at intermediate $k$ to greater values at large and small $k$. Ranges of $k$ where the peaks of $|\operatorname{Im}(\omega) / \operatorname{Re}(\omega)|$ exceed some threshold (which is $\ll 1$ ) correspond very closely with ranges of $k$ where the numerical LH mode breaks up into a series of ion Bernstein modes.

Relativistic effects are not included in either analytic or numerical work and we need to check that they are not important. Although the speeds involved are low, for waves very close to perpendicular propagation weakly relativistic effects are necessarily important even for what would normally be considered non-relativistic speeds (Robinson 1987).

In summary, our analytic dispersion relation for LH waves agrees far better with our numerical results than any previous dispersion relation over a large range of plasma parameters and wavenumbers. The agreement is extremely good when ion magnetization effects are small. However, when ion magnetization effects become larger, the numerical and analytic results differ qualitatively, with no numerical solution being found at frequencies very close to multiples of $\Omega_{i}$ where the ion magnetization effects are greatest.

\section{References}

Bingham, R., Dawson, J. M., \& Shapiro, V. D. 2002, J. Plasma Phys., 68, 161

Cairns, I. H. 2001, Publ. Astron. Soc. Aust., 18, 336

Cairns, I. H. \& Zank, G. P. 2002, Geophys. Res. Lett., 29, 1143

Feng, W., Gurnett, D. A., \& Cairns, I. H. 1992 J. Geophys. Res., 97, 17005

Melrose, D. B. 1986 Instabilities in space and laboratory plasmas (Cambridge: Cambridge University Press)

Omel'chenko, Yu. A., Sagdeev, R. Z., Shapiro, V. D., \& Shevchenko, V. I. 1989, Sov. J. Plasma Phys., 15, 427

Robinson, P. A. 1987, Phys. Fluids, 31, 107

Willes, A. J. \& Cairns, I. H. 2000, Phys. Plasmas, 70, 3167 